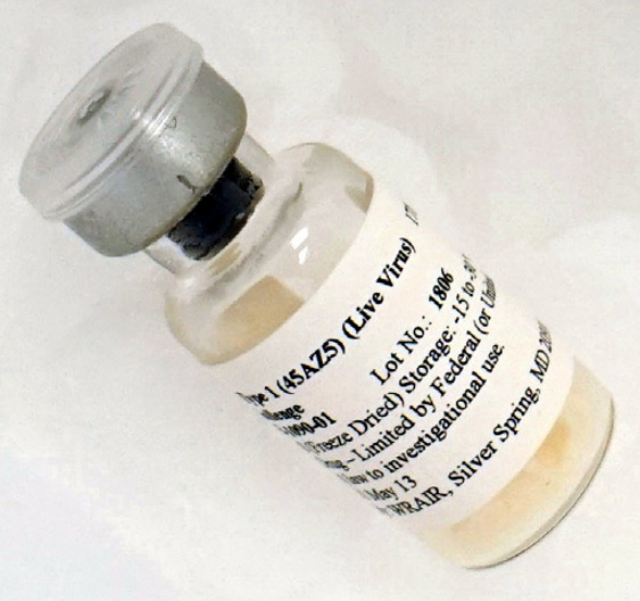

\title{
Challenge accepted: Human challenge trials for dengue
}

\author{
By Wudan Yan
}

In 1973, Duane Gubler learned the hard way that mosquitoes loaded with dengue virus might choose to bite humans over monkeys. Gubler, now retired after studying dengue virus for over 45 years, is a world dengue expert. In the 1970s, he was working at University of Hawaii School of Medicine in Honolulu, investigating how much dengue virus in the blood of an animal could infect its natural host, the mosquito Aedes aegypti. As Gubler was testing this question in monkeys, he first had to get the animals sick.

Instead of directly injecting the monkeys with the virus by needle, Gubler let dengue-infested mosquitos feed on the monkeys and infect them with dengue naturally. The infected mosquitos were contained in a pint-sized cylindrical carton with both ends first hollowed and covered in fine mesh. After he brought the monkeys out of their cages to be infected, Gubler placed the mesh ends of the carton close to the belly of each monkey so that the mosquitos could bite the animal. "On some days, I had a hard time getting the mosquitos to feed on the monkey, so I would put my hand between the monkey's belly and the mesh covering of the mosquitos' carton to entice the mosquitos to probe the monkey. But one of them actually probed me," he says. A few days later, when Gubler fell severely ill, he knew he had dengue. Gubler asked his colleague, Robert Tesh, to have the mosquitos feed on Gubler's arm so that his viremia-the level of virus in his blood-could be measured. "I infected all the mosquitos that fed on me: I had over 8 logs of virus, which correlates with pretty severe disease, circulating for over six days," says Gubler. "I was one sick puppy."

Although Gubler accidentally got dengue, researchers now want to deliberately infect humans directly with dengue virus in what are called human challenge studies, with a new study of this kind slated to begin recruitment at the end of this month. These experiments can help elucidate how an infectious agent uniquely causes illness in humans over in other animals. Ultimately, these experiments would help scientists develop better interventions-either vaccines or treatments-for specific diseases. To date, no vaccines or therapies exist for dengue, a disease that has increased in incidence by more than 30 -fold in the last 50 years, according to the World Health Organization (WHO). With more than one-third of the world's population living in dengue-endemic regions, as many as 400 million people per year require medical attention after infection and onset of a fever. Without proper medical care, patients can progress to hemorrhagic fever and septic shock, and they can potentially die. A dengue human infection model (DHIM) would help scientists delineate the progression of disease in humans and ultimately design better vaccines and drugs against the virus.

Harris Graham, a pathologist from the American College in Syria, performed the first dengue human infections with live, unattenuated virus in the early 1900s (Med. Rec. New York, 61, 204-207, 1902). Albert Sabin, better known for his work in developing an oral polio vaccine, followed up on Graham's early experiments after the rise in dengue among US troops during World War II (Am. J. Trop. Med. Hyg., 1, 30-50, 1952). The results from these experiments have shaped much of what the scientific and medical communities now know about dengue: the virus's incubation period, the clinical features of dengue, the four subtypes of dengue virus, and the transient and variable immunity to secondary infections. According to Gubler, no subjects from these early experiments died after being infected with live, unattenuated virus. "But there was nothing ethical about these early experiments," he says. "These studies were simply done because dengue became a public health concern." 


\section{Remodeling dengue}

After the second set of human challenge experiments in the 1940s and 1950s, researchers wondered whether humans could be spared by using other animals, such as mice and nonhuman primates, to study dengue. However, by the 1990s, scientists realized that these animal models do not adequately recapitulate the disease in humans, and they started to look into resurrecting the DHIM.

In the late 1990s, researchers at the Walter Reed Army Institute of Research in Silver Spring, Maryland, took previously developed vaccines for dengue off the shelf and studied their effects in humans. These vaccines-live attenuated dengue viruses- had never made it to market because they produced too many symptoms of disease to be taken through the entire vaccine development process, explains Mark Polhemus, an infectious disease researcher at State University of New York (SUNY) Upstate Medical University in Syracuse, who was not involved in the Walter Reed trials. "But that's what makes them perfect for a controlled human infection model," he says. Two of those formerly shelved vaccines were found to be suitable for human challenge models because they are able to cause a mild dengue-like fever in healthy volunteers (Vaccine, 32, 1488-1494, 2014).

A separate human challenge trial also completed by Walter Reed researchers in 2002 used attenuated dengue virus strains that could cause dengue-like fever in people previously vaccinated against all four dengue subtypes ( $J$. Infect. Dis., 207, 700-708, 2013). Vaccinated volunteers, should, in theory, be protected from subsequent dengue infections. Yet this wasn't the case: vaccination against all four subtypes of dengue offered variable protection when the volunteers were challenged with different subtypes of dengue virus. This study revealed that human challenge models could provide useful and essential information to drug companies looking to develop vaccines against dengue: many vaccine developers believe that for a vaccine to be successful, it would have to adequately prevent infection from all four subtypes.

From these recent proof-of-concept experiments that demonstrated that human infection with attenuated dengue viruses could be done safely, the SUNY Institutional Review Board and the US Army Medical Research and Material Command Human Research Protection Office approved, on 28 May and 8 June, respectively, a dengue human challenge trial that will offer a more detailed examination of the disease in humans. The US Food and Drug Administration (FDA) gave permission for the trial to proceed on 15 May. The trial coordinators, Polhemus and Timothy Endy, both at SUNY Upstate, are hoping to recruit healthy volunteers starting at the end of July.

Not only will this new, in-patient trial help establish the optimal dose of dengue virus-1 (DENV-1) to cause symptoms of disease with the fewest adverse effects for use in subsequent human challenges, but it will also be the first human dengue challenge to track changes in the volunteers' immune systems using gene sequencing and proteomic tools. Despite the prevalence of dengue, much remains unknown about how the disease progresses in humans. The upcoming trial will be the first of its kind in providing an in-depth look at how the immune system reacts to dengue infection using RNA-sequencing and proteomics tools. "There's an enormous amount of science to be gleaned from this study," Polhemus says.

"We'll be able to pick apart their immune response daily to see how that virus is acting," Endy says. Because dengue has a latency period of approximately 4-10 days, patients usually do not arrive in the clinic until after this window has passed and they have come down with disease. How a person's immune system reacts during this latency period is largely unknown; by looking at the gene and biomarkers on a regular basis after a controlled human challenge, scientists can look at the different phases of virus infection in close detail. Scientists also wonder whether certain genes can protect people from becoming severely ill: although some people become intensely febrile, others hardly experience any symptoms of disease. In the controlled experimental setting, the doctors will know each patient's medical history and immunological status prior to infection.

\section{Scrutinizing protection}

Dengue virus infection inhibits the innate immune response by blocking type 1 interferon signaling in human dendritic cells, which are known to turn on the adaptive immune response (J. Virol., 84, 4845-4850, 2010). The human challenge experiment for dengue completed by the researchers at Walter Reed in 2002 found that interferon- $\gamma$, a type 2 interferon with roles in both innate and adaptive immunity, seems to protect volunteers from developing fever and viremia (Vaccine, 29, 3895-3904, 2011).

One of the unique aspects of dengue is that there are four serotypes (DENV-1, DENV-2, DENV-3 and DENV-4), which confounds the issue of whether people infected with one type can be protected from subsequent infections. "The fact that there are four serotypes of dengue adds a layer of complexity we don't see with HIV, or most other viruses," Endy says. A person infected with DENV-1, for example, will develop antibodies against DENV-1, and is thought to have lifelong protection against DENV-1, but will not be protected against infection with any of the other three virus serotypes. Instead, it is thought that pre-existing antibodies against the virus can physically latch onto a different subtype of dengue (for example, DENV-2) during a secondary infection and help the DENV-2 virus enter cells, in a phenomenon scientists call 'antibody-dependent enhancement'.

"It's hard to say if the antibodies produced against the virus can correlate with protection," Endy says. With the new trial, however, researchers will be able to examine the immunological changes in healthy volunteers from infection to convalescence, and compare the gene and protein profiles of people challenged with virus to that of those who receive a placebo.

Clues from studying infected individuals living in dengue-endemic regions of the world have instructed dengue immunologists to investigate the mechanisms more deeply in other animal models. In humans, different human leukocyte antigen (HLA) alleles, which encode proteins that help the immune system recognize foreign invaders like viruses and bacteria, help mediate susceptibility or protection to dengue fever. The HLA complex turns on the adaptive, T cell response in dengue infection (Proc. Natl. Acad. Sci., 110, E2046-E2053, 2013).

"It's absolutely crucial to include a good T cell response in preventing dengue infection," says Sujan Shresta, an immunologist at the La Jolla Institute for Allergy and Immunology in La Jolla, California. Shresta's lab group found that the T cell response could override the antibodydependent enhancement effects in mouse models (J. Immunol., 193, 4117-4124, 2014). "The human challenge studies can really help us figure out what's happening at the molecular and cellular level that mediate a good $\mathrm{T}$ cell response," she says. "Then, we can explore the mechanisms more deeply in mice."

\section{Vaccine development}

In addition to opening up a host of interesting questions about the immunology of dengue, 
the upcoming DHIM studies can also aid in the development of a dengue vaccine.

Currently, there are six vaccine candidates for dengue, one of which just completed phase 3 trials in dengue-endemic countries (N. Engl. J. Med., 372, 113-123, 2015; Lancet, 384, $1358-1365,2014)$. Whereas vaccines should usually confer $80-90 \%$ protection, the dengue vaccine that has moved furthest along in clinical trials, developed by Sanofi-Pasteur, was only approximately $60 \%$ effective in protecting a total of more than 30,000 children against symptomatic dengue.

"The Sanofi vaccine didn't do so well, and we don't know why. With the challenge model, we can take volunteers who have been vaccinated and challenge them selectivity with each virus and pick apart-in what I would call a forensic analysis-why the vaccine performed the way it did in field studies," Endy says.

"If a vaccine candidate cannot induce an immune response in a healthy volunteer that can protect them from a human challenge strain, then it's very unlikely that the vaccine can protect them from a wild-type strain delivered from a mosquito," says Col. Stephen Thomas at the Walter Reed Army Institute of Research. "A human infection can be very informative in distinguishing vaccine candidates that have a chance of succeeding in the field versus those that won't."

Nicholas Jackson, head of research and development for dengue vaccine program at Sanofi Pasteur in Lyon, France, agrees. "A DHIM model would be useful after phase 1 trials in the scope of vaccine development. It would provide justification for the candidate before embarking on the lengthier phase 2 and phase 3 studies," he says. Although Sanofi Pasteur developed its vaccine without human challenge models for dengue, the field trials were still informative. "The phase $2 \mathrm{~b}$ and phase 3 efficacy trials have provided important insight into how our vaccine works up against the complexity and unpredictable nature of dengue. Our trial results suggest that our vaccine could halve dengue disease of any severity and to significantly reduce severe forms of the disease."

The human challenge model for malaria, optimized in 1986, has been essential for modern-day vaccine development (Am. J. Trop. Med. Hyg., 35, 66-68, 1986). As of January 2015 , there are more than 20 vaccine candidates for malaria in development, according to the WHO. Human challenges for malaria vaccines are often done concurrently with phase 1 and $2 b$ studies, which investigate the safety and efficacy of a candidate, respectively, according to Ashley Birkett, director of the Program for Appropriate Technology in Health's (PATH) Malaria Vaccine Initiative program, based in Seattle.
"With these challenge models that are so powerful, we tend to move through vaccines and kill things quite quickly that don't hit the mark from the challenge model," Birkett says. "That's been really effective in terms of accelerating development and not spending lots of resources on a vaccine that's not going anywhere."

In addition to human challenges with malaria, similar experiments with influenza in the 1960s and 1990s helped develop many of the first-line antivirals for flu, such as amantadine (Symmetrel) and oselatmivir (Tamiflu), according to Matthew Memoli, an infectious disease researcher at the National Institute of Allergy and Infectious Diseases in Bethesda, Maryland. These human challenge models for influenza seem to track with the development of new therapies against flu. "No influenza human challenges were done from 2001 until 2012, and consequently, no novel class of influenza antivirals was approved in that time," Memoli says.

In contrast with malaria and influenza, a human challenge model does not yet exist for pertussis, a disease that could be better understood using such models. Better known as whooping cough, pertussis is a respiratory disease caused by the bacterium Bordetella pertussis. Despite the fact that an estimated $84 \%$ of babies worldwide are vaccinated against pertussis, the incidence of disease is still rising; one of the challenges
"These studies can be, in many ways, controversial." for dengue, and they aim to perform such experiments ethically. "When you look at the ethical framework of human infections, you need to ask: are there non-human models available and have we adequately explored what those models could be?" Thomas asks. "I think the answer we've arrived to for dengue is: yes, we have explored non-human models, and no, there are no other better models." Yet despite the wealth of information human challenges can provide, scientists must design these experiments, first and foremost, with the volunteers' safety in mind.

Researchers who have looked at human challenge models concur on the importance of maintaining the safety of these trials. "The key for human challenge experiments is to have a specific question you want answered," says Anna Durbin, a dengue vaccine expert at the Johns Hopkins Bloomberg School of Public Health in Baltimore. "From that, you can decide, 'what endpoints do I need to answer that question'? Our idea should not be to cause severe disease in any volunteers." In the upcoming trial, for example, the challenge with dengue- 1 is intended to cause fever and viremia so the researchers can study what happens to the immune system after infection.

With these considerations in mind, scientists are able to design ethical and safe human challenge models for dengue in order to investigate questions that have confounded them for decades. Four decades with pertussis is to develop a vaccine that will not be reactive in children, and that will protect not only against the onset of disease, but also against infection. So far, no such vaccine exists. "Pertussis is a uniquely human pathogen," says Tod Merkel, a microbiologist at the FDA's Center for Biologics. "The field of pertussis research and vaccine development could benefit greatly from a human challenge model for the disease."

\section{Safety first}

Human challenge experiments are ethically unique because none of them are intended to benefit the subject. "Therefore, these studies can be, in many ways, controversial," says Arthur Caplan, a bioethicist at New York University. "In these dengue human challenge trials, most of the ethical weight is on the institutional review board. They have the objectivity to look at the merit of the science and the risks involved. The volunteer subjects can't do that."

Dengue researchers seem to agree on the importance of having a human model ago, Gubler and his colleague, Lyon Rosen, asked the US National Institutes of Health to perform human trials, but those trials never took off: Gubler soon moved to Indonesia-he now lives in Utah-and there were no other researchers who were interested in these studies. Reinstating these human challenges could address many questions Gubler has had for years.

"I'm sort of a maverick: I don't believe that we need a balanced tetravalent vaccine for dengue because I don't think antibodydependent enhancement is the most important component of determining whether a person gets severe disease or not," Gubler says. "It's important, but there are factors that I think are equally or perhaps more important. There's so much we don't understand about the immunology, virology, and the host-virus interactions that cause severe disease, which only underscores the importance of human challenge studies."

Wudan Yan is a freelance science journalist and a former news intern at Nature Medicine. 